\title{
DIAGNÓSTICO AMBIENTAL DE LA CANTERA YARAYABO PROVINCIA SANTIAGO DE CUBA, CUBA
}

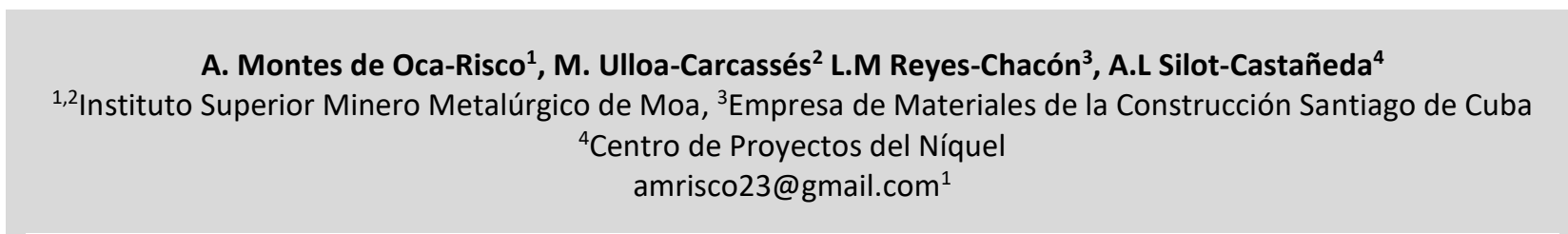

Recepción 23/12/2017 - Aprobación 17/01/2018

DOI: $10.15628 /$ holos. 2018.6728

\section{RESUMEN}

La extracción de áridos es una de las actividades que mayor impacto genera al medio ambiente. En la actualidad la provincia de Santiago de Cuba tiene concesionados 8 yacimientos donde se explotan recursos destinados a la producción de materiales para la construcción. Las áreas degradadas por la explotación de áridos en la provincia ocupan alrededor de 200 ha, lo cual provoca un gran impacto al medio ambiente. Por tal razón se propuso como Objetivo: realizar una evaluación de impacto ambiental de la explotación de la cantera Yarayabo, con el fin de proponer las medidas preventivas y correctoras que habrán de adoptarse para la minimización del impacto sobre el entorno, a fin de compatibilizar la explotación y la preservación del medio natural. En la metodología: se aplicaron métodos científicos que permitieron el análisis de los factores que influyen en la alteración del medio ambiente producto de la explotación minera. Alcanzando como resultado: la matriz de impacto ambiental de la cantera objeto de estudio. Y como conclusión: se propusieron medidas para cada una de las componentes impactadas para mitigar sus afectaciones y lograr así una minería responsable.

PALABRAS-CLAVE: evaluación de Impacto Ambiental, canteras de áridos, diagnóstico ambiental, minería responsable

\section{ENVIRONMENTAL DIAGNOSIS OF THE YARAYABO QUARRY PROVINCE SANTIAGO DE CUBA, CUBA}

\begin{abstract}
The extraction of aggregates is one of the activities that generates the greatest impact on the environment. Currently, the province of Santiago de Cuba has 8 reservoirs where resources are exploited for the production of materials for construction. The areas degraded by the exploitation of aggregates in the province occupy around 200 ha, which causes a great impact on the environment. For this reason, it was proposed as Objective: to carry out an environmental impact assessment of the exploitation of the Yarayabo quarry, in order to propose the preventive and
\end{abstract}

corrective measures that must be adopted to minimize the impact on the environment, in order to make the exploitation and preservation of the natural environment. In the methodology: scientific methods were applied that allowed the analysis of the factors that influence the alteration of the environment resulting from mining exploitation. Reaching as result: the environmental impact matrix of the quarry object of study. And in conclusion: measures were proposed for each of the components impacted to mitigate their effects and thus achieve responsible mining.

KEYWORDS: environmental impact Assessment, aggregate quarries, environmental diagnosis, responsible mining 


\section{INTRODUCCIÓN}

La minería es una actividad necesaria, ya que si se quiere desarrollar la agricultura (tan necesaria en sí misma) o la pesca, si se quiere vivir en ciudades y no en cavernas; se necesitan herramientas, materias primas y aditivos para el suelo, que hagan posible la realización de estas actividades humanas.

La explotación de canteras a cielo abierto comprende una parte importante de la minería a nivel mundial, su objetivo principal es el suministro de materiales para la construcción, ya sean áridos o rocas ornamentales. Este tipo de minería se caracteriza por generar serias alteraciones medioambientales, debido a que la mayoría de las empresas, no prestan la debida atención a dicha actividad. La intensidad de las mismas, depende de varios factores, entre los que se reconocen la situación y morfología del yacimiento, así como las características del entorno. Estas influencias, van transformando el paisaje y provocan el empeoramiento cualitativo o cuantitativo, de los recursos y el origen o desarrollo de procesos dañinos o degradantes. De aquí, la necesidad de conocer los diferentes aspectos necesarios para la correcta explotación de una cantera.

En Cuba, el desarrollo de la actividad minera, se ha intensificado en los últimos años, sobre todo la explotación de canteras de materiales para la construcción como en la explotación de minerales metálicos, producto a la creciente demanda de nuevas obras de construcción desde el punto de vista técnico, infraestructural y social en todo el país.

La provincia Santiago de Cuba no ha estado ajena a este desarrollo minero en la industria de Materiales de la Construcción, que va aparejado con la aparición de nuevos impactos ambientales y la intensificación de los ya existentes, elementos estos que requieren de una caracterización minero-ambiental para minimizar los impactos al medio ambiente (Pérez, 2015).

Como situación problémica de esta investigación se plantea que en la cantera Yarayabo, el concesionario no cuenta con un plan para rehabilitar las posibles afectaciones ambientales, al estudiar el proyecto de explotación de la cantera, en el mismo solamente se hace alusión a las reposiciones de carácter natural del mineral a extraer.

Una aproximación a esta situación problémica demostró la existencia del siguiente Problema de la investigación: La necesidad de realizar una evaluación de impacto ambiental en la cantera Yarayabo, para minimizar su impacto sobre el entorno.

Con vista a la solución de este problema se propone como objetivo de la investigación, realizar una evaluación de impacto ambiental de la explotación de la cantera Yarayabo, con el fin de proponer las medidas preventivas y correctoras que habrán de adoptarse para la minimización del impacto sobre el entorno, a fin de compatibilizar la explotación y la preservación del medio natural.

\section{REVISIÓN BIBLIOGRÁFICA}

\subsection{Minería, Medio ambiente y Evaluación de Impacto Ambiental}

Según la Norma ISO 14001, Medio Ambiente está definido como: "es el entorno en el cual una organización opera, incluyendo el aire, el agua, la tierra, los recursos naturales, la flora, la fauna, los seres humanos y sus interrelaciones", donde "el entorno en este contexto se extiende desde el interior de una organización hasta el sistema global". 
Otra definición dice que Medio Ambiente "es el sistema de elementos abióticos, bióticos y socioeconómicos con que interactúa el hombre, a la vez que se adapta al mismo, lo transforma y lo utiliza para satisfacer sus necesidades" (Ley No.81, 1997).

Se denomina Evaluación de Impacto Ambiental (EIA) al procedimiento técnico administrativo que sirve para identificar, prevenir e interpretar los impactos ambientales que producirá un proyecto en su entorno en caso de ser ejecutado, con el fin de que la administración competente pueda aceptarlo, rechazarlo o modificarlo. Se dice que hay impacto ambiental cuando una acción o actividad produce una alteración, favorable o desfavorable, en el medio o en alguno de los componentes del medio. Esta acción puede ser proyecto de ingeniería, un programa, un plan, una ley o una disposición administrativa con implicaciones ambientales. Hay que hacer constar que el término impacto no implica negatividad, ya que éstos pueden ser tanto positivos como negativos (Conesa, 1997).

La evaluación del impacto ambiental surge a fines de los años 60 en Estados Unidos con el nombre de Environmental Impact Assessment en algunos casos en lugar de Assessment se puede encontrar Analysis o Statement. EI EIA introduce las primeras formas de control de las interacciones de las intervenciones humanas con el ambiente (ya sea en forma directa o indirecta), mediante instrumentos $\mathrm{y}$ procedimientos dirigidos a prever $\mathrm{y}$ evaluar las consecuencias de determinadas intervenciones. Todo esto con la intención de reducir, mitigar, corregir y compensar los impactos (Dávila, 2014).

El impacto ambiental sobre el medio ambiente es la diferencia entre la situación del medio ambiente futuro modificado, tal y como se manifestaría como consecuencia de la realización del proyecto y la situación del medio ambiente futuro tal como habría evolucionado normalmente sin tal actuación, es decir, la alteración neta (positiva o negativa en la calidad de vida del ser humano) resultante de una actuación.

Desde el punto de vista del medio ambiente, las operaciones extractivas suscitan inquietudes de dos tipos. En primer lugar, la utilización de recursos en sí no renovables puede suponer el agotamiento de los mismos para las generaciones futuras. En segundo lugar, el impacto de la explotación minera puede dañar la calidad del medio ambiente. El impacto ambiental de las operaciones extractivas incluye aspectos como la contaminación atmosférica (principalmente por el polvo), el ruido, la contaminación del agua o del suelo y diversas repercusiones en los niveles freáticos, la destrucción o perturbación de hábitats naturales y el impacto visual en el paisaje circundante (Herrera, 2008).

\subsection{Análisis y Revisión Bibliográfica}

En los últimos años son numerosas las investigaciones realizadas a nivel internacional acerca de la evaluación de impacto ambiental en la minería.

Conesa (1997), ofrece procedimientos y métodos de trabajo que permiten captar el enfoque integral que requiere un trabajo de protección ambiental, propone el uso de métodos cualitativos y cuantitativos, las matrices y una tipología de clasificación de 10 clases de impactos. En su matriz de importancia relaciona las acciones, tanto en la fase constructiva como en la fase de funcionamiento del proyecto y los factores del medio que pueden ser afectados por dichas acciones, se propone una metodología detallada para las evaluaciones de impactos.

Uno de los trabajos más completo e integral que trata sobre la temática corresponde al Manual de restauración de terrenos y evaluación de impactos ambientales en minería del España, Carcedo, F. \& Fernández (1989), donde se abordan diversos temas relacionados con la actividad minera y el medio ambiente, desde los tipos de explotaciones mineras, hasta la preparación de los terrenos para efectuar la revegetación. En el mismo, además de analizarse los 
principales procesos que generan degradación de los suelos, establece algunos métodos de evaluación y medidas de mitigación.

Por otra parte, Hernández et al. (2011) determinó el impacto ambiental que produce la explotación del yacimiento de calizas El Cacao, la metodología permitió identificar los impactos ambientales producidos por las acciones de desbroce, destape y extracción, perforación y voladura, excavaciones, transportación, procesamiento de la materia prima y su almacenamiento; así como la interacción entre estas acciones y los componentes del medio (suelo, aire, agua, flora, fauna, paisaje, infraestructura y economía). Se encontró que los impactos más considerables ocurren durante el desbroce y destape, y que los componentes ambientales más afectados son el suelo, el aire, el agua y la economía. Finalmente se proponen medidas correctoras para mitigar las afectaciones ambientales.

Por su parte, Handjaba (2012) determina como la extracción de los materiales de la construcción del yacimiento El Cacao, en la provincia Granma, Cuba, provoca impactos ambientales al entorno de esta cantera. Para conocer estas alteraciones y las medidas para mitigarlas utilizó un procedimiento metodológico que permitió identificar las acciones mineras, tales como componentes impactantes del medio ambiente y factores ambientales susceptibles a recibir impactos, y a través de su interacción se identificó, caracterizó, valoró y evaluó los impactos ambientales. Se establecieron las premisas para lograr que la cantera El Cacao realice una operación minera respetuosa con el medio ambiente.

Khabali \& Targuisti (2013) realizan una EIA de las canteras en la región de Kenitra. Su propósito es asegurar que las canteras activas, abandonadas o las que se inaugurarán en un futuro no causará ningún daño significativo o irreversible al medio ambiente local y/o regional. Este estudio se diseñó para identificar los riesgos potenciales que suponen y los efectos globales causados las explotaciones en su conjunto.

Finalmente, Gallardo et al. (2015), en su investigación presenta la evaluación de los impactos ambientales provocados por la actividad minera en la mina Santa Lucía Castellanos, ubicada al noroeste de la provincia de Pinar del Río, Cuba. Para la identificación y evaluación de los impactos se utilizó la matriz causa-efecto, matriz de Leopold (1971), que permitió establecer las medidas de mitigación, seguimiento y control de los impactos negativos generados por esta actividad en la región.

\subsection{Marco legal relacionado con la evaluación minero ambiental en el mundo y Cuba}

Las normas que protegen indirectamente al medio ambiente surgen ya desde la Revolución Industrial, las primeras normas específicamente ambientales surgen en los países más desarrollados.

Los primeros órganos administrativos ambientales se crean a fines de los '60 en países desarrollados, esto incide en otros países. En los '70 en la conferencia de Estocolmo y con el informe del Club de Roma, se acuerda la necesidad urgente de responder al problema del deterioro ambiental y se postula el desarrollo sustentable. En los '80 se reunió por primera vez la Comisión Mundial sobre Medio Ambiente y Desarrollo, conceptúa como sostenible el modelo de desarrollo que "atiende a las necesidades del presente sin comprometer la posibilidad de que las futuras generaciones atiendan a sus propias necesidades". En los '90 en Río de Janeiro, la conferencia de las Naciones Unidas sobre medio ambiente se aprueba el Convenio sobre diversidad biológica "Agenda 21", la Conferencia fue la oportunidad de adoptar un programa de acción para el siglo XXI relacionado entre otros aspectos con la protección del medio ambiente.

Después de la primera cumbre histórica de Río de Janeiro en 1992 y diez años después de la de Johannesburgo en 2002. Se realizó la Cumbre de la Tierra Río+20, llamada oficialmente 
Conferencia de Naciones Unidas sobre Desarrollo Sustentable. Esta cumbre fue un nuevo intento de Naciones Unidas en el comienzo de milenio para avanzar sobre el compromiso de los Estados y la comunidad mundial en los grandes cambios de este siglo XXI.

En el caso de Cuba, se presta especial atención a la protección del medio ambiente en el contexto de una política de desarrollo. La protección del medio ambiente contribuye a asegurar la disponibilidad de los recursos naturales indispensables para la satisfacción de las necesidades básicas de la población. La Ley No. 76 de Minas aprobada en 1995, establece la política minera y las regulaciones jurídicas de dicha actividad. Además traza directivas obligatorias, controladas por los funcionarios de gobierno vinculados con la actividad. Dicha ley establece en su artículo 41 que "todos los concesionarios están obligados a preservar adecuadamente el medio ambiente y las condiciones ecológicas del área, elaborando estudios de impactos y planes para prevenir, mitigar, controlar, rehabilitar y compensar el impacto derivado de la actividad minera en los términos que establece la legislación".

Como instrumento legal para regir la política ambiental en Cuba, se aprueba la Ley No. 81 de Medio Ambiente en 1997, complementada con el Decreto Ley No. 200, de las Contravenciones en materia de medio ambiente de 1999. Esta ley establece que: "los organismos que tienen a su cargo el uso y administración de recursos naturales, en cumplimiento de sus deberes, atribuciones y funciones específicas relativas a la protección del medio ambiente, deben incorporar y evaluar los requerimientos de la protección del medio ambiente en sus políticas, planes y programas de desarrollo y ejecutar proyectos con vista a garantizar la sostenibilidad de su gestión y contribuir al desarrollo de la vida en un medio ambiente adecuado, valorando científicamente los factores ambientales."

\section{METOdOLOGÍA}

La Empresa de Materiales para la Construcción de Santiago de Cuba en la actualidad tiene concesionados 8 yacimientos, los cuales están destinados a satisfacer las crecientes necesidades que se imponen en toda la provincia de materiales para la construcción. Algunos yacimientos se explotan hace más de 30 años con la finalidad de obtener materia prima para la construcción. En la provincia las áreas afectadas por esta actividad minera ocupan más de 200 ha. Montes de Oca \& Ulloa (2013).

\subsection{Caracterización del área de estudio}

Ubicación del área de estudio

El yacimiento de calizas Yarayabo se encuentra ubicado en el municipio de Palma Soriano (Figura 1), en la provincia Santiago de Cuba. Está aproximadamente a unos $6 \mathrm{~km}$ al Sur de la ciudad de Palma Soriano.

Las coordenadas geográficas del yacimiento son:

$20^{\circ} 09^{\prime} 01^{\prime \prime} \mathrm{N}$

$75^{\circ} 58^{\prime} 45^{\prime \prime} \mathrm{O}$ 


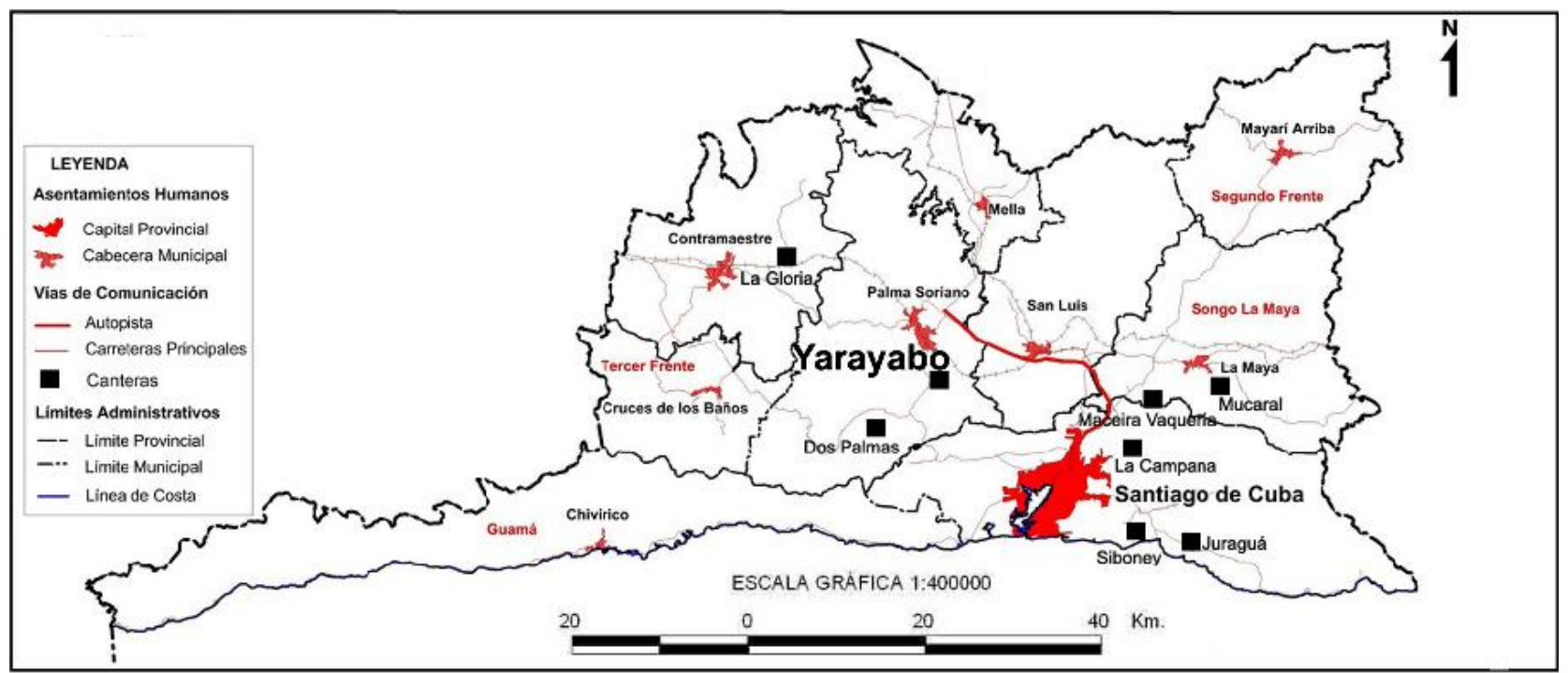

Figura 1: Ubicación de la cantera Yarayabo.

Relieve

El relieve del área está constituido por colinas de gradientes suaves que alcanzan alturas entre 200 y 300 m sobre el nivel del mar. Las mayores alturas se encuentran localizadas en la parte sur del yacimiento y las menores en la parte norte.

\section{Clima}

La temperatura media anual está entre 22 y $24^{\circ} \mathrm{C}$. Las precipitaciones medias anuales son de $600 \mathrm{~mm}$.

\section{Comunicación}

Las comunicaciones se realizan por la carretera central, importante arteria asfaltada que une en estos casos la ciudad de Santiago de Cuba y Palma Soriano, la autopista que une ambas ciudades, la carretera de Palma a Cueto y otros de menos importancia como la que une a Palma Soriano y San Luis. Por la ciudad de Palma Soriano pasa la vía férrea del circuito Sur del Oriente. El yacimiento se comunica con la carretera central mediante un terraplén semi-asfaltado.

\section{Economía de la región}

La importancia económica de la región radica en la agricultura. Ésta se extiende a grandes plantaciones de café, caña y en menos cantidad frutos menores. Además existen tres centrales azucareros, dos importantes centrales de población, Palma Soriano y Palmarito del Cauto además se encuentran pequeños poblados (Hatillo, Yarayabo y Perú).

\section{Geología y tectónica del yacimiento}

El yacimiento se encuentra ubicado en la región oriental en la subregión llanura del Cauto al Sureste de Palma Soriano. Las rocas que conforman el yacimiento corresponden a la formación Puerto Boniato del complejo de cuencas superpuestas del arco volcánico Paleógeno, constituida por calizas fragmentarias órgano-detríticas y calizas pelitomórficas abundantes en $\mathrm{SiO}_{3}$ (pedernal) en forma de nódulos que constituyen la materia prima fundamental del yacimiento. La edad de esta formación es del Eoceno Medio.

En el yacimiento existen elementos estructurales poco desarrollados y frecuentes, limitándose a representar un pliegue artificial post sedimentario. Este pliegue es un fenómeno local, generalmente las capas buzan hacia el NO. Los elementos tectónicos están poco 
desarrollados en el yacimiento. Los sectores del yacimiento están separados por una zona en la cual existe gran acumulación de materia arcillosa con fragmentos de roca y que constituye un límite natural entre dos zonas; ésta geomorfológicamente es una cañada.

\section{Características hidrogeológicas del yacimiento}

El río más cercano al yacimiento es el Yarayabo, pero no afecta directamente al mismo, se encuentra a $2.5 \mathrm{~km}$ de distancia, este es un afluente del río Cauto, la arteria principal que drena la región. Específicamente en el área que abarca al yacimiento se presentan algunas cañadas.

Flora y fauna

La flora de la zona se encuentra muy afectada por la acción del hombre debido a los impactos que ocurren en las áreas de extracción de la cantera y en las áreas incididas por las escombreras. La fauna que se observa en la concesión minera representa el remanente de la que originalmente poblaba esta zona y no se caracteriza por un alto valor endémico.

En la Tabla 1 se puede observar los elementos de la flora y la fauna que más abundan en el entorno de la cantera.

Tabla 1: Representantes de la flora y la fauna en la cantera Yarayabo.

\begin{tabular}{llll}
\hline \multicolumn{2}{c}{ Representantes de la fauna } & \multicolumn{2}{c}{ Representantes de la flora } \\
\hline Nombre común & Nombre científico & Nombre común & \multicolumn{1}{c}{ Nombre científico } \\
\hline Jutía & Capromyidae & Maguey & Agave \\
Aura tiñosa & Catharter aura & Guao & Comocladia dentata \\
Rana toro & Lithobates catesbeianus & Cupey & Clusia rosea \\
Lagartija verde & Teius teyou & Mango & Mangifera indica \\
Sinsonte & Mimus polyglottos & Palma & Arecaceae \\
Totí & Ptiloxena atroviolacea & Coco & Cocos nucifera \\
Zunzún & Chlorostilbon ricordii & Malagueta & Xylopia obtusifolia \\
Majá & Epicrates anzulyso & Marabú & Dichrostachys glomerata \\
\hline
\end{tabular}

\section{Elementos generales del laboreo minero}

Los elementos principales de la explotación se muestran en la Tabla 2, el sistema de explotación que se utiliza es el arranque con perforación y voladura, el reapile se realiza mediante el uso del buldózer Chetra T-20, la carga se efectúa con el cargador frontal XCMG - LW$800 \mathrm{~K}$ con capacidad de carga de $5 \mathrm{~m}^{3}$ y el transporte en camiones de volteo Kraz con una capacidad de carga de $8 \mathrm{~m}^{3}$. La explotación se realiza de este a oeste mediante escalones de 10$12 \mathrm{~m}$ (Figura 2). 
Tabla 2: Elementos principales de la explotación.

\begin{tabular}{lc}
\hline \multicolumn{1}{c}{ Factores } & Dimensiones \\
\hline Altura del escalón $(\mathrm{m})$ & 25 \\
Talud del escalón en su estado de trabajo & 75 \\
(grados) & 65 \\
Talud del escalón en su estado final (grados) & 5 \\
Ancho de la berma de seguridad $(\mathrm{m})$ & \\
\hline
\end{tabular}

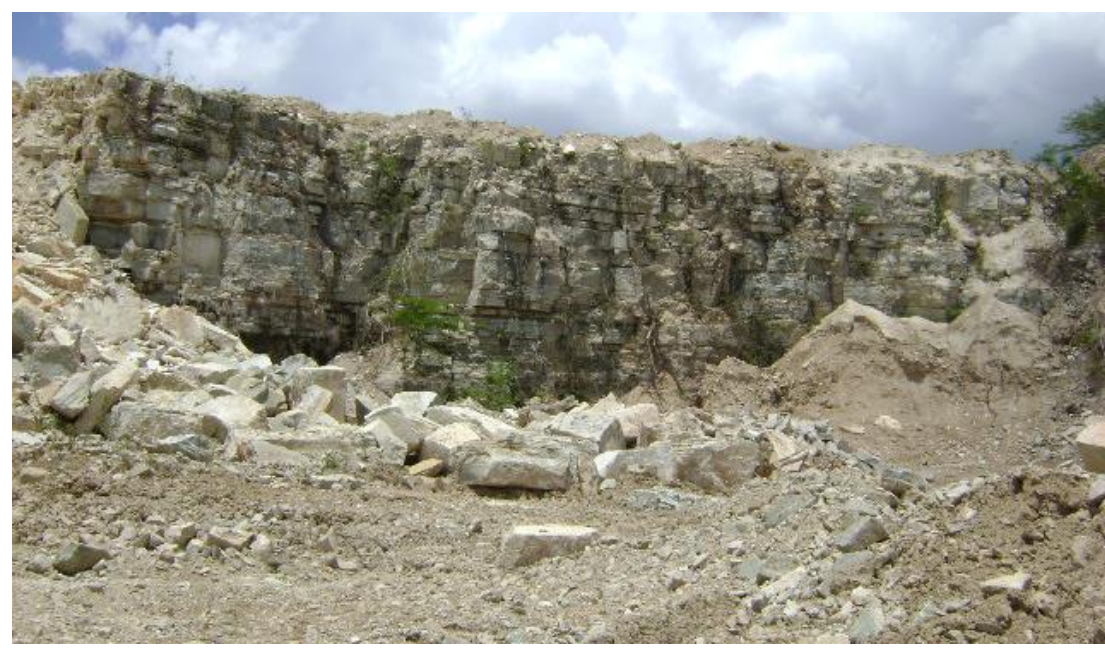

Figura 2: Escalones de trabajo en la cantera Yarayabo.

\section{Escombreras}

En el yacimiento existen dos escombreras (Figura 3), las cuales están situadas fuera de los límites de las reservas geológicas y dentro del área concesionada, aprovechando también el relieve de la zona. La escombrera No. 1 (E1) está ubicada al SW, en la cual se está depositando el estéril que se está extrayendo del área donde se está realizando el destape. A medida que avanza el desarrollo hacia la parte norte, está ubicada otra escombrera, ya que la distancia de tiro se hace mayor, por lo que la escombrera No.2 (E2) se encuentra al NW del yacimiento.

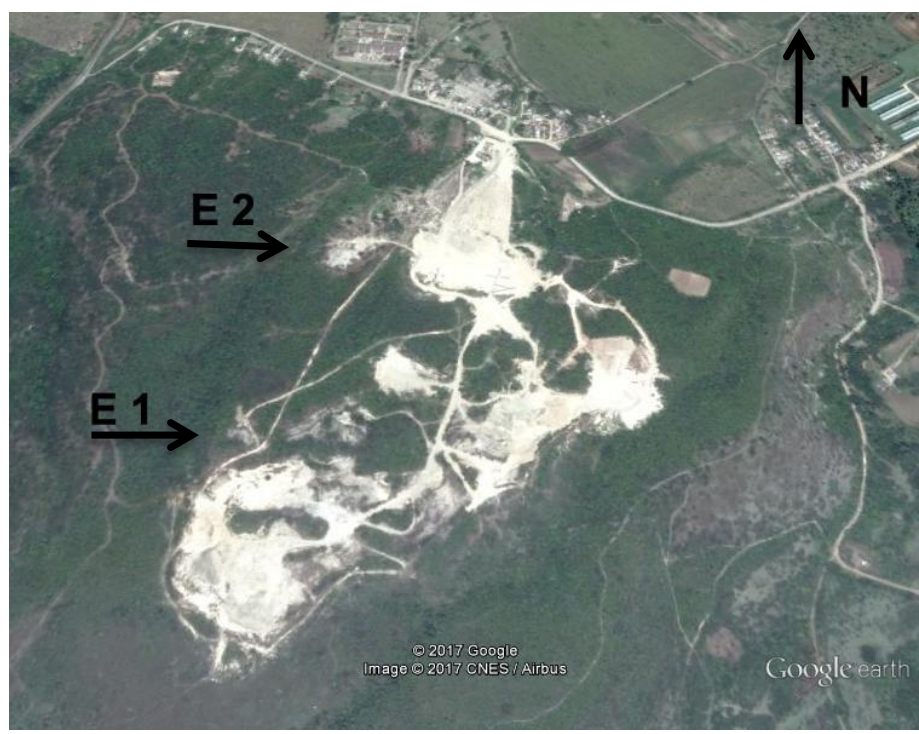

Figura 3: Ubicación de las escombreras en la cantera Yarayabo. 


\section{Descripción medio ambiental de la zona}

La explotación mineral en este tipo de yacimiento y el procesamiento de la materia prima generan impactos ambientales en el medio:

El impacto a la atmósfera va estar representado principalmente por la contaminación por la emisión de polvo producto de la perforación (Figura 4), creación de escombreras, tráficos de equipos (Figura 5) y trituración de la materia prima. Contaminación por la emisión de gases tóxicos como consecuencia del laboreo minero y construcción de escombreras. Contaminación por emisión de ruidos de los equipos tecnológicos, no tecnológicos y las voladuras (Figura 6).

La contaminación de las aguas superficiales se ve afectada por partículas sólidas y elementos tóxicos disueltos tales como: restos de materiales explosivos, combustibles y lubricantes.

Como resultado de la explotación del yacimiento, el paisaje natural original se ve afectado por la interacción del hombre donde sus acciones han modificado las propiedades del mismo (Figura 7).

El suelo es afectado por el desarrollo de los procesos erosivos y acumulativos como consecuencia del laboreo minero, además de la pérdida de la capa vegetal y la compactación.

La flora y la fauna son uno de los factores más fuertemente impactados, especialmente por la deforestación (Figura 8) y la desaparición de especies endémicas.

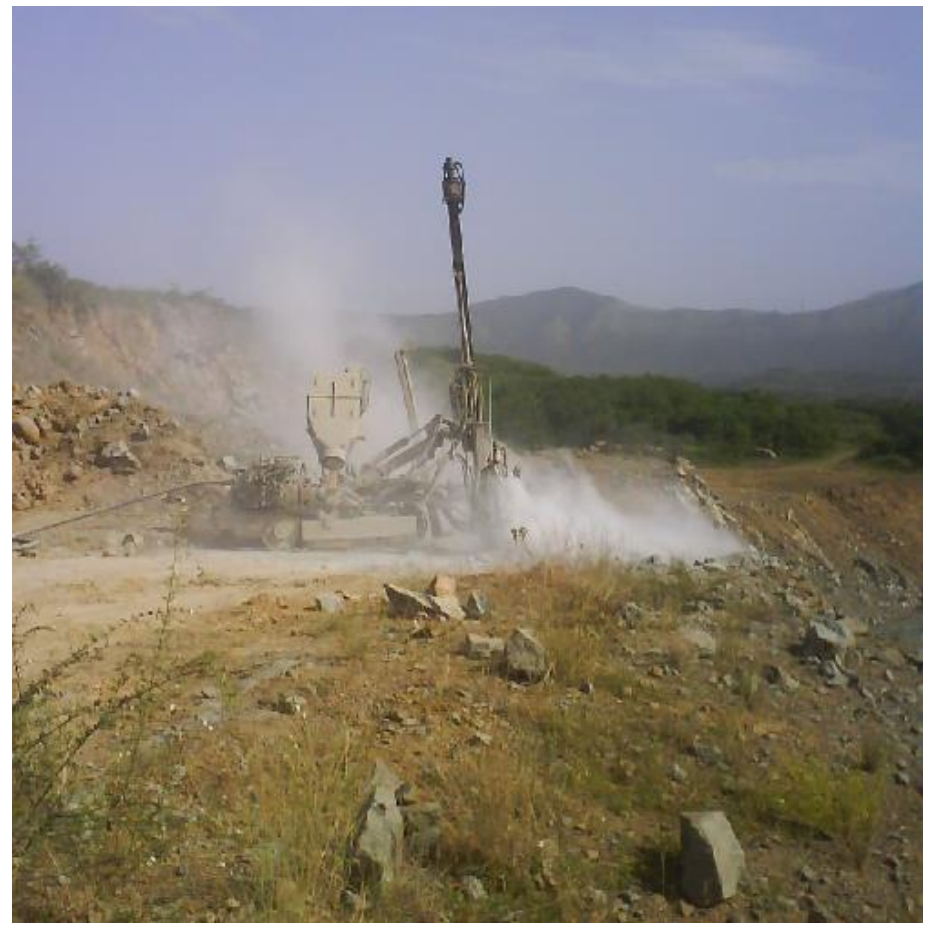

Figura 4: Impacto del polvo producto de la perforación de taladros 


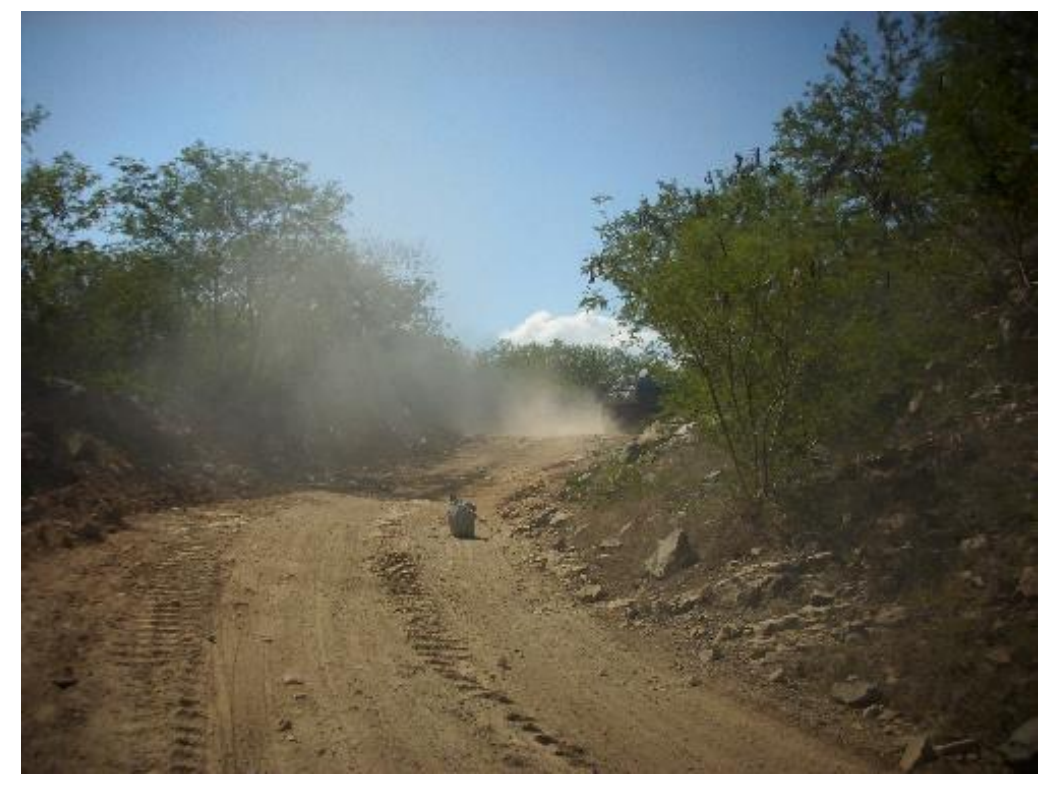

Figura 5: Impacto del polvo producto del tráfico de camiones

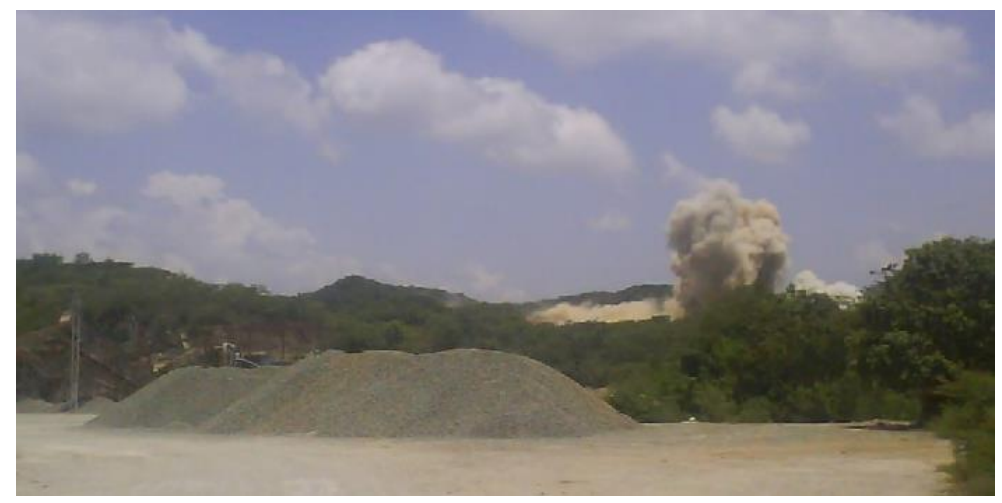

Figura 6: Impacto de la voladura al medio ambiente

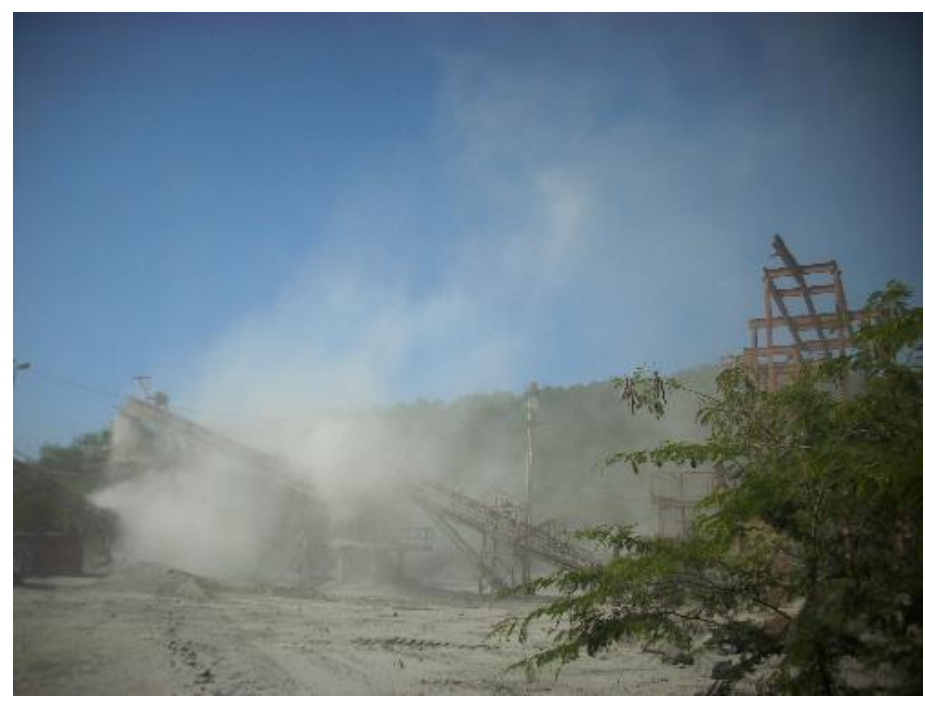

Figura 7: Impacto al paisaje producido por el polvo de las plantas de trituración 


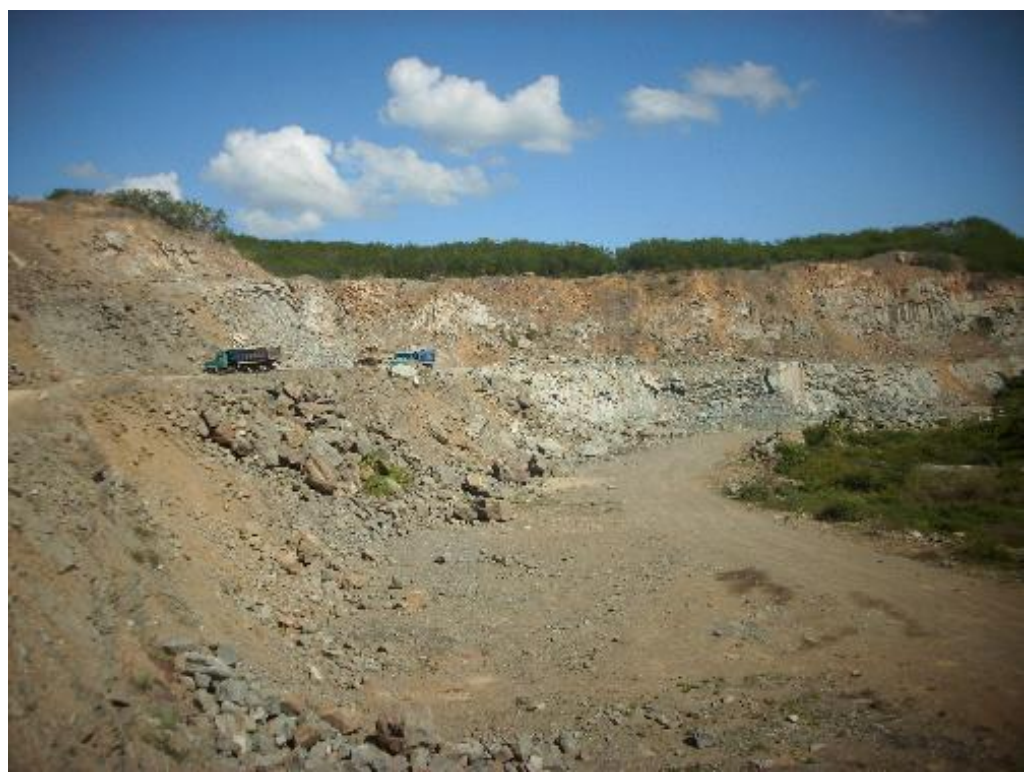

Figura 8: Deforestación del área de la cantera producto de la explotación minera

\subsection{Metodología para la evaluación de los impactos ambientales en la cantera}

Para la identificación, caracterización y evaluación de los impactos, se utilizó la Metodología para la Realización de las Solicitudes de Licencia Ambiental y los Estudios de Impacto Ambiental, propuestas por el Ministerio de Ciencia Tecnología y Medio Ambiente y el Centro de Inspección y Control Ambiental (CICA) de Cuba, en su versión vigente del 2009, adaptadas a las condiciones específicas del objeto de investigación y la referencia del trabajo de evaluación de impactos de (García, 2010). Utilizando la observación directa, consultas de expertos, escenarios comparados, tormentas de ideas, las encuestas y entrevistas. El impacto total se calculó según la expresión (1)

$$
\mathrm{I} T=M g+\mathrm{I}+\mathrm{S}+\mathrm{R}+\mathrm{O}+\mathrm{D}+\mathrm{C}
$$

Donde: Mg Magnitud, I Importancia, S Sinergismo, R Reversibilidad, O Ocurrencia, D Duración, C Certeza. Estos indicadores alcanzan un valor máximo de 10 puntos que luego se multiplicará por los siete indicadores para un total de 70 puntos total, que finalmente se promedia para ubicarlos en cada una de las categorías propuestas en términos de: compatibles, moderados, severos y críticos.

\section{RESULTADOS Y DISCUSIÓN}

Las acciones que pueden causar impactos ambientales son: En la fase de exploración geológica (Tabla 3), en la fase de explotación minera (Tabla 4) y los factores del medio susceptibles a recibir impactos (Tabla 5). 
Tabla 3: Acciones que pueden causar impacto ambiental en la fase de exploración geológica.

\begin{tabular}{cc}
\hline (a) & Construcción de trochas y caminos \\
\hline (b) & Perforación de pozos de exploración y toma de \\
muestras
\end{tabular}

Tabla 4: Acciones que pueden causar impacto ambiental en la fase de explotación minera.

\begin{tabular}{cc}
\hline (d) & Desbroce \\
\hline (e) & Preparación de los frentes de trabajo \\
\hline (f) & Perforación de la roca \\
\hline (g) & Carga y voladura de la roca \\
\hline (h) & Formación de escombreras \\
\hline (i) & Almacenamiento del material útil \\
\hline (j) & Carga del material útil \\
\hline (k) & Transporte del material útil \\
\hline
\end{tabular}

Tabla 5: Factores del medio susceptibles a recibir impactos.

\begin{tabular}{cl}
\hline & I. Clima \\
Medio físico & II. Hidrografía \\
& III. Suelo \\
& IV. Paisaje \\
\hline \multirow{2}{*}{ Medio biótico } & V. Flora \\
& VI. Fauna \\
\hline \multirow{2}{*}{ Medio socioeconómico } & VII. Población \\
& VIII. Sociales y culturales \\
\hline
\end{tabular}

Se identificaron los impactos siguientes:

\section{A la atmósfera:}

(1) Incremento en el nivel de ruidos y vibraciones

(2) Disminución de la calidad atmosférica por emisiones de gases, polvo y partículas (Figura 5 y 6 ).

(3) Modificaciones al nivel microclimático, como consecuencia de la eliminación de la vegetación.

\section{Hidrografía}

(4) Contaminación por combustibles y lubricantes.

(5) Alteración de la calidad del agua subterránea por variación en la infiltración.

(6) Modificación del nivel piezométrico.

III. Suelo

(7) Pérdida o alteración del suelo fértil por operaciones de excavación, construcción de caminos, acopio de material y escombreras.

(8) Aumento de la erosión y sedimentación. 

IV. Al paisaje y la morfología:
(9) Modificación de las características visuales del paisaje.
(10) Cambios en la morfología.
(11) Disminución del atractivo paisajístico.
V. A la flora:
(12) Reducción de especies ocasionada por la tala de árboles.
(13) Pérdida de la vegetación en general

\section{A la fauna}

(14). Pérdida de unas especies y colonización del espacio por otras de menor calidad.

(15). Desplazamiento de los animales hacia otros medios similares.

\section{A la población:}

(16) Incremento del nivel de empleo.

(17) Mejoramiento de la red de transporte.

(18) Mejoramiento de las comunicaciones.

\section{Sociales y culturales:}

(19) Incremento en el número de accidentes y enfermedades.

(20) Enriquecimiento de la diversidad cultural.

Teniendo en cuenta los factores ambientales y las operaciones impactantes se elaboró la tabla de identificación de los impactos ambientales en las diferentes etapas de la minería en la cantera Yarayabo (Tabla 6).

La evaluación del impacto total tuvo como finalidad llegar a clasificar los impactos ambientales en términos de:

Impacto Compatible (IP): Son los que tienen muy poca entidad, si su efecto es perjudicial al cesar las causas que lo producen, en poco tiempo se restablecen las condiciones medio ambientales, originales, con el concurso de los procesos regenadores. Se clasifican en leve, local, directo, primario, reversible, sin sinergias, se recupera a corto plazo, poco probable y presenta un valor menor que 18 de impacto total.

Impacto Moderado (IM): Produce daños de poca magnitud, pero su importancia comienza a ser considerable. Tras el mismo las condiciones físicas originales se restablecen con el solo concurso de los mecanismos naturales del medio, aunque la recuperación es larga. Se clasifica en leve, regional en cierto grado, primario, reversible con dificultad, sin sinergias aparentes, se recupera a mediano plazo y es probable, alcanza un valor de 18-29 de impacto total.

Impacto Severo (IS): Se trata de impactos de magnitud notable y de gran importancia, cuando cesa la causa que lo origina, la recuperación de las condiciones iniciales del medio, se hace muy difícil y requiere a veces de la adopción y puesta en prácticas de medidas correctoras de sus efectos. Se clasifica con moderada intensidad, regional, indirecto o secundario, reversible con dificultad y medidas correctoras, con algunas sinergias, poco importantes, muy probable que se produzca, alcanza un valor de impacto total entre 30 y 35. 
Tabla 6: Identificación de los impactos en la etapa de exploración geológica y explotación minera.

\begin{tabular}{|c|c|c|c|c|c|c|c|c|c|c|c|c|}
\hline \multirow{3}{*}{\multicolumn{2}{|c|}{$\begin{array}{c}\text { Factores } \\
\text { ambientales }\end{array}$}} & \multicolumn{11}{|c|}{ Operaciones impactantes } \\
\hline & & \multicolumn{3}{|c|}{ Fase Exploración Geológica } & \multicolumn{8}{|c|}{ Fase Explotación Minera } \\
\hline & & $\mathrm{a}$ & $b$ & c & $d$ & e & $f$ & g & $\mathrm{h}$ & $\mathrm{i}$ & j & k \\
\hline \multirow{4}{*}{$\begin{array}{l}\text { Medio } \\
\text { físico }\end{array}$} & 1 & $1,2,3$ & 1,2 & $1,2,3$ & $1,2,3$ & $1,2,3$ & 1,2 & $1,2,3$ & 1,2 & 1,2 & 1,2 & $1,2,3$ \\
\hline & II & 4 & 5,6 & 4,5 & 4,5 & 4 & 4,5 & 5 & 5 & 4 & - & $4,5,6$ \\
\hline & III & 7,8 & 7 & 7,8 & 7,8 & 7,8 & 8 & 7,8 & 7,8 & 7,8 & 7 & 7,8 \\
\hline & IV & $9,10,11$ & - & - & $9,10,11$ & $9,10,11$ & 9 & $9,10,11$ & $9,10,11$ & $9,10,11$ & 10 & 9 \\
\hline \multirow{2}{*}{$\begin{array}{l}\text { Medio } \\
\text { biótico }\end{array}$} & V & 12,13 & 12 & 12,13 & 12,13 & 13 & - & 13 & 12,13 & - & - & 13 \\
\hline & VI & 14,15 & 15 & 14,15 & 14,15 & 14,15 & 14 & $\begin{array}{l}14, \\
15 \\
\end{array}$ & 14,15 & - & - & 14,15 \\
\hline \multirow{2}{*}{$\begin{array}{c}\text { Medio } \\
\text { socio- } \\
\text { económico }\end{array}$} & VII & 16,17 & 16 & 16 & $\begin{array}{l}16, \\
17\end{array}$ & 16 & 16 & 16 & - & 16 & - & $\begin{array}{c}16,17 \\
, 18\end{array}$ \\
\hline & VIII & 19 & 19 & 19 & 19 & 19 & 19 & 19 & 19 & - & 19 & 19,20 \\
\hline
\end{tabular}

Impacto Crítico (IC): Es el impacto que por su enorme magnitud e importancia, supera el denominado Umbral del Impacto, que es el límite a partir del cual se considera que el deterioro el irreversible, la acción capaza de producirlo provoca la destrucción completa de elementos o recursos naturales que son piezas claves en el funcionamiento del paisaje, imponiendo en su lugar una dinámica regresiva continuamente adversa al establecimiento de las condiciones que posibilitarán su restitución. Se clasifica en fuerte, global o regional, acumulativo, irreversible, a largo plazo se mantiene, con sinergias importantes o catastróficas, inevitable y el impacto total alcanza un valor mayor que 35 .

Tabla 7: Escala de clasificación de los impactos del CITMA (2008).

\begin{tabular}{cc}
\hline RANGO & CLASIFICACIÓN \\
\hline IT $<18$ & IMPACTO COMPATIBLE (IP) \\
\hline IT $18-29$ & IMPACTO MODERADO (IM) \\
\hline IT $30-35$ & IMPACTO SEVERO/ MUY BENÉFICO (IS) \\
\hline IT $>35$ & IMPACTO CRÍTICO/ EXTREMADAMENTE BENÉFICO (IC) \\
\hline
\end{tabular}

Después de la determinación de los impactos totales producidos (Tabla 6) sobre los factores ambientales se realiza la valoración de cada impacto (Tabla 9) teniendo en cuenta los indicadores propuestos por el (Ministerio de Ciencia Tecnología y Medio Ambiente, 1999) y la ponderación de los impactos (Tabla 8). 
Tabla 8: Ponderación de los impactos.

\begin{tabular}{|c|c|}
\hline Naturaleza ( $\mathrm{Na})$ & Positivo (+) / Negativo (-) \\
\hline \multirow{3}{*}{ Magnitud (Mg) } & Leve: $1,2,3$ \\
\hline & Moderado: 4, 5, 6 \\
\hline & Fuerte: $7,8,9,10$ \\
\hline \multirow{4}{*}{ Importancia (I) } & Local: 1, 2, 3 \\
\hline & Zonal: 3, 4, 5 \\
\hline & Regional: 6, 7, 8 \\
\hline & Global: 9, 10 \\
\hline \multirow{4}{*}{ Sinergismo (S) } & Sin sinergias aparentes: 1 \\
\hline & Con sinergias poco importantes: $2,3,4$ \\
\hline & Con sinergias importantes: 5, 6, 7 \\
\hline & Con sinergia catastrófica: 8, 9, 10 \\
\hline \multirow{3}{*}{ Reversibilidad (R) } & Reversible: 1,2 \\
\hline & Reversible con medidas: $3,4,5,6,7$ \\
\hline & Irreversible: $8,9,10$ \\
\hline \multirow{3}{*}{ Ocurrencia (O) } & Directos o primarios: $1,2,3$ \\
\hline & Indirectos o secundarios: $4,5,6$ \\
\hline & Acumulativos: $7,8,9,10$ \\
\hline \multirow{3}{*}{ Duración (D) } & Corto plazo: $1,2,3$ \\
\hline & Mediano plazo: 4, 5, 6 \\
\hline & Largo plazo: 7, 8, 9, 10 \\
\hline \multirow{3}{*}{ Certeza (C) } & Poco probable: $1,2,3$ \\
\hline & Probable: 4, 5, 6 \\
\hline & Inevitable: 7, 8, 9, 10 \\
\hline
\end{tabular}

Tabla 9: Matriz de evaluación de los impactos.

\begin{tabular}{|c|c|c|c|c|c|c|c|c|c|c|}
\hline \multirow{2}{*}{ Impactos } & \multicolumn{8}{|c|}{ Indicadores } & \multirow{2}{*}{$\begin{array}{l}\text { Ponderación } \\
\text { de los Impactos }\end{array}$} & \multirow{2}{*}{ IT } \\
\hline & $\mathrm{Na}$ & $\mathrm{Mg}$ & I & $S$ & $\mathrm{R}$ & 0 & $\mathrm{D}$ & C & & \\
\hline 1 & - & 6 & 6 & 4 & 2 & 4 & 2 & 7 & 23 & $\mathrm{IM}$ \\
\hline 2 & - & 5 & 4 & 5 & 4 & 3 & 3 & 6 & 30 & IS \\
\hline 3 & - & 4 & 2 & 3 & 5 & 5 & 5 & 4 & 28 & $\mathrm{IM}$ \\
\hline 4 & - & 3 & 3 & 2 & 5 & 3 & 3 & 4 & 23 & $\mathrm{IM}$ \\
\hline 5 & - & 4 & 3 & 3 & 8 & 7 & 3 & 3 & 31 & IS \\
\hline 6 & - & 5 & 3 & 2 & 5 & 6 & 7 & 1 & 29 & $\mathrm{IM}$ \\
\hline 7 & - & 7 & 5 & 6 & 9 & 8 & 8 & 10 & 53 & IC \\
\hline 8 & - & 4 & 4 & 5 & 5 & 5 & 4 & 7 & 34 & IS \\
\hline 9 & - & 4 & 4 & 5 & 5 & 6 & 3 & 7 & 34 & IS \\
\hline 10 & - & 7 & 5 & 6 & 10 & 5 & 8 & 10 & 50 & IC \\
\hline 11 & - & 4 & 5 & 5 & 5 & 7 & 2 & 8 & 36 & IC \\
\hline 12 & - & 8 & 5 & 6 & 6 & 5 & 5 & 9 & 43 & IC \\
\hline 13 & - & 8 & 5 & 6 & 5 & 6 & 8 & 8 & 46 & IC \\
\hline 14 & - & 6 & 3 & 6 & 5 & 5 & 3 & 6 & 34 & IS \\
\hline
\end{tabular}




\begin{tabular}{lllllllllll}
\hline 15 & - & 5 & 3 & 5 & 6 & 6 & 4 & 8 & 37 & $\mathrm{IC}$ \\
\hline 16 & + & 1 & 4 & 3 & 2 & 3 & 6 & 7 & 26 & $\mathrm{IM}$ \\
\hline 17 & + & 1 & 3 & 3 & 1 & 3 & 7 & 7 & 25 & $\mathrm{IM}$ \\
\hline 18 & + & 1 & 3 & 3 & 1 & 3 & 7 & 6 & 24 & $\mathrm{IM}$ \\
\hline 19 & - & 5 & 3 & 5 & 3 & 2 & 2 & 5 & 25 & $\mathrm{IM}$ \\
\hline 20 & + & 1 & 2 & 3 & 1 & 3 & 7 & 6 & 23 & $\mathrm{IM}$ \\
\hline
\end{tabular}

\subsection{Análisis y ponderación de los impactos}

Del análisis de la valoración de cada uno de los impactos reflejado en la (Tabla 9), teniendo en cuenta los indicadores propuestos en su ponderación. Se obtuvo un total de 20 impactos, de ellos solo cuatro se clasificaron como positivos (Incremento del nivel de empleo, Mejoramiento de la red de transporte, Mejoramiento de las comunicaciones y Enriquecimiento de la diversidad cultural), el resto es de naturaleza negativa.

Finalmente se obtuvo como resultado la siguiente (Figura 9) donde se puede observar que los impactos que superan al 35 son impactos críticos, es el caso del 7, 10, 11, 12, 13 y 15.

4.2 Medidas preventivas, correctoras y de mitigación para los componentes del medio más afectados

Para establecer las medidas preventivas, correctoras y de mitigación, nos auxiliamos en lo expuesto por Vadillo (1994).

I. Protección a la atmósfera: (1) Instalación de dispositivos adecuados de eliminación de la contaminación (filtros) en todo el equipamiento que funciona con diesel y con gasolina, plantas de preparación mecánica por vía seca y cerciorarse que estos funcionen adecuadamente. (2) Colocar durante la perforación filtros de manga, que permitan la captación directa del polvo. (3) Pavimentación de accesos permanentes a la cantera. (4) Revegetación de terrenos restituidos (superficies finales de vertederos y taludes excavados). (5) Control del polvo durante la perforación por medio de captadores y reducción del número de tajos con voladuras. (6) Empleo de pantallas vegetales o artificiales contra el viento, que dificultan su libre circulación en los niveles superficiales. (7) Estudiar rutas alternativas de transporte en zonas próximas a las áreas habitadas. (8) Utilizar explosiones con microrretardos para disminuir la actividad sísmica. (9) Emplear silenciadores en motores y plantas. (10) Disminuir hasta $10 \mathrm{~m}$ la altura del frente de explotación. (11) Presentar un calendario de mantenimiento anual, con la obligación de cumplir lo programado por las maquinarias y herramientas mineras. (12) Realizar un mantenimiento preventivo adecuado y recubrir de goma los elementos metálicos que sufren los impactos de las rocas.

II. Protección a la hidrografía: (13) Creación de sistemas de drenaje, generales para la recogida de las aguas externas a la zona y particulares para cada escombrera y taludes. (14) Deberán recogerse los aceites usados tras el mantenimiento de la maquinaria, si este se realiza en la zona de explotación. (15) Aislamiento de materiales fácilmente disgregables y contaminantes, cubriendo con otros materiales procedentes de la propia explotación. (16) Revegetación de áreas restituidas y reducción de la superficie afectada por las labores mineras, ambas acciones para disminuir la erosión. (17) Establecimiento de un sistema de análisis periódicos que detecte las variaciones y anomalías inadmisibles en las características del agua, a la salida de la planta y antes de su entrada en el receptor. (18) Adecuación de la planta de tratamiento más conforme a las características de los contaminantes del agua (depuración, balsa de decantación, filtros en drenajes, etc). 


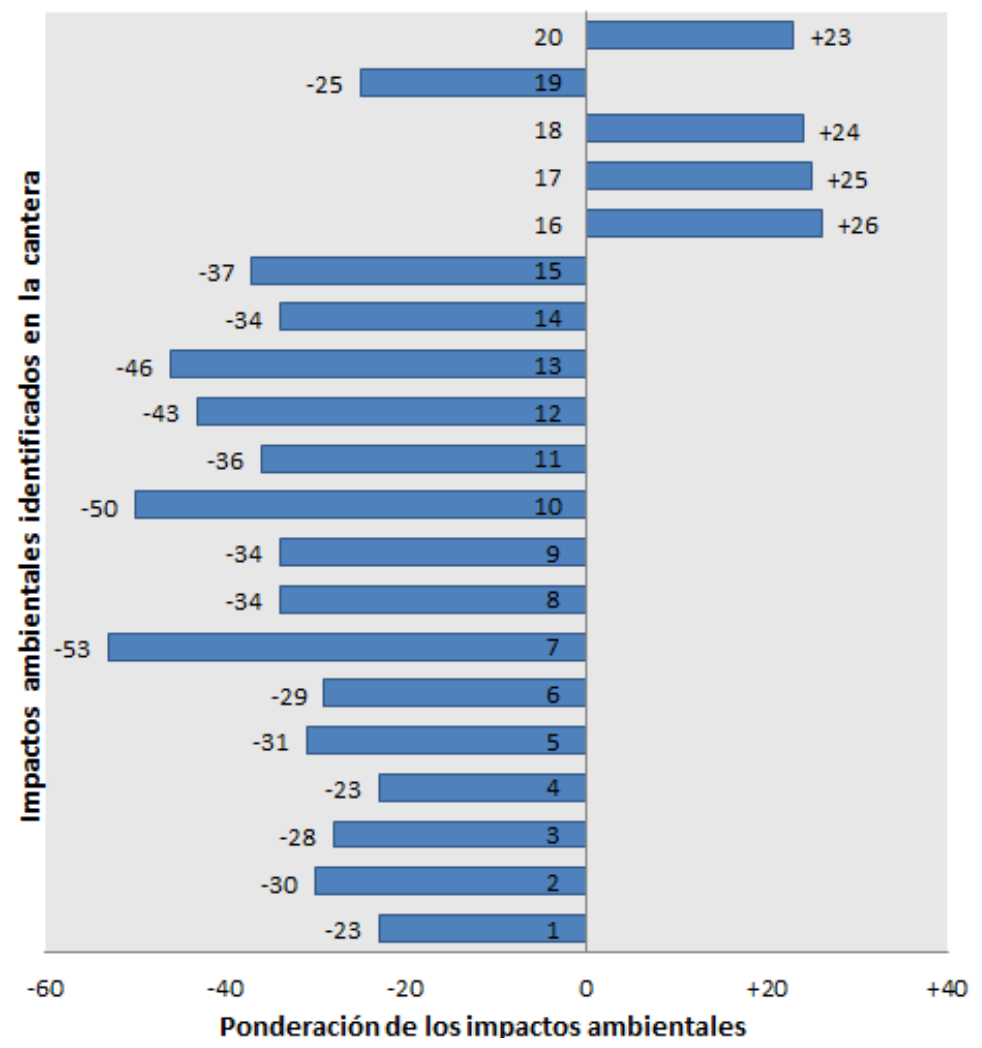

Figura 9: Gráfico de ponderación de los impactos.

III. Protección al suelo (19) Diseño de un modelado en la recuperación que permita la utilización productiva y ecológica del terreno una vez explotado. (20) Extraer la capa de suelo antes de realizar el destape en las zonas con una capa superficial de escombro y darle un uso apropiado. (21) Utilizar los escombros en la lucha contra la erosión y emplear gaviones en los lugares más críticos para evitar la erosión y el derrumbe de las riberas. (22) Evitar ubicaciones de escombrera en zona con peligro de hundimiento.

IV. Protección al paisaje: (23) Plantación de árboles y arbustos que actúen como pantallas visuales. (24) Remodelar la topografía alterada de modo que se ajuste lo más posible a lo natural. Utilizar los productos de las excavaciones para rellenar en otros lugares. (25) Buscar un uso alternativo de los estériles y materiales no aprovechables.

V. Protección a la flora y fauna: (26) Reducir al mínimo las áreas por desbrozar. (27) Promover la revegetación entre las organizaciones sociales y de masa de los pobladores de la zona, con ayuda de la empresa minera y de sus trabajadores, e impulsar un movimiento de recuperación de plantas endémicas. (28) Propiciar con medidas complementarias el retorno de los representantes de la fauna del territorio. (29) Mantener áreas de preservación permanente. (30) Adoptar un cronograma de deforestación ajustado con el avance del frente de explotación, evitando la remoción anticipada de vegetación. (31) Recuperación progresiva de las áreas afectadas, usando especies nativas. (32) Revegetación compensatoria en áreas anteriormente degradadas por otras causas, de forma de compensar la pérdida de hábitats por la reconstrucción de un hábitat equivalente en términos de estructura y extensión; como esta medida sólo surte efecto a largo plazo, debe iniciarse lo más temprano posible. (33) Evitar la formación de islas de vegetación demasiado pequeñas para asegurar la viabilidad de las poblaciones faunísticas. (34) Conectar las áreas de vegetación remanentes por medio de corredores y conectar las áreas bajo influencia directa de la obra con otras manchas de vegetación existentes en los alrededores. 
VI. Protección a la población: (35) Disposición de carteles indicadores de peligro. (36) Ubicar las tomas de agua para uso y consumo de la población fuera de la zona de influencia de la extracción, y facilitar a la comunidad afectada los medios para la instalación y acopio de agua potable. (37) Apoyar la implementación de un programa de educación para la salud respecto al agua de consumo.

\section{CONCLUSIONES}

En el cantera Yarayabo concesionada a la Empresa de Materiales de la Construcción de Santiago de Cuba se realizó la evaluación de los impactos ambientales que se producen durante la explotación de roca caliza a partir del establecimiento de las principales acciones susceptibles de producir dichos impactos (desbroce, perforación, carga y voladura del mineral, carga y transportación, formación de escombreras y su almacenamiento) y su interacción con los componentes del medio (suelo, aire, agua, flora, fauna, paisaje y la población). Todas las acciones del proyecto afectan en mayor o menor medida a todos los factores del medio. Los impactos más significativos ocurren en la etapa de desbroce y la extracción; los componentes del medio más afectado son: el suelo, el paisaje, la flora y la fauna. Para cada una de las componentes impactadas se propusieron medidas para mitigar sus afectaciones y lograr así una minería responsable.

\section{REFERENCIAS}

Carcedo, F. J. \& Fernández, L. V. (1989). Manual de restauración de terrenos y evaluación de impactos ambientales en minería. ITGE/MTE. Madrid, pp. 321.

CICA (2009). Guía para la realización de la Solicitudes de Licencia Ambiental y los estudios de Impactos Ambiental. La Habana. Centro de Inspección y Control Ambiental.

Conesa, F. (1997). Metodología para la evaluación del impacto ambiental. Madrid, España: Editorial Mundi - Prensa.

Dávila, D. A. (2014). Procedimientos sobre el estudio de impacto ambiental (EIA) en la urbanización hacienda de las flores municipio de Villa Nueva, República de Guatemala (tesis de pregrado). Universidad de San Carlos de Guatemala, Guatemala.

Decreto Ley No.200. (1999). De las contravenciones en materia de medio ambiente. Gaceta Oficial de la República de Cuba.

Gallardo, D., Bruguera, N., Díaz, J. \& Cabrera, I. (2015) Impacto provocado por la minería en la zona de Santa Lucía: evaluación físico-química. Minería y Geología. 31(4), pp. 100-120

García, S. (2010) Evaluación de impacto ambiental de la construcción de túneles populares en el municipio Moa (Tesis de maestría). Instituto Superior Minero Metalúrgico de Moa, Cuba.

Handjaba, H. J. (2012). Estudio minero-ambiental de la cantera El Cacao. Ciencia \& Futuro, 2(4), pp. 49-58.

Hernández, N., Ulloa, M. \& Rosario, Y. (2011). Impacto ambiental de la explotación del yacimiento de materiales de construcción El Cacao. Minería y Geología. 27(1), pp. 38-53.

Herrera, J. (2008). La protección medio ambiental en minería y el desarrollo minero sostenible. Universidad Politécnica de Madrid. España.

Khabali, H. \& Targuisti, K. (2013). Explotación de canteras en la costa de Kenitra y su área de influencia: estudio de impacto ambiental. Revista de Estudios Andaluces. 30(1), pp. 1 -26.

Leopold, L. B. (1971). A procedure for evaluating environmental impact. Geol. Surv. USA. Washington D.C. 
Ley No. 76 de Minas. (1995). Gaceta Oficial de la República de Cuba.

Ley No.81, Ley de Medio Ambiente. (1997). Gaceta Oficial de la República de Cuba.

Montes de Oca, A. \& Ulloa, M. (2013) Recuperación de áreas dañadas por la minería en la cantera Los Guaos, Santiago de Cuba, Cuba. Revista Lunazul. 37, pp. 74-88.

Pérez, A. (2015) Caracterización Minero-Ambiental de las Canteras en la Industria de Materiales de la Construcción de Santiago de Cuba (Tesis de grado). Instituto Superior Minero Metalúrgico de Moa, Cuba.

Resolución No. 77/99 (1999). "Reglamento del proceso de evaluación de Impacto Ambiental". Ministerio de Ciencia, tecnología y Medio Ambiente de Cuba.

Vadillo, F. (1994). Problemática ambiental de la minería de los áridos naturales. Tecnoambiente. 13 , pp. $47-50$. 